\title{
Properties of Quadratic Flux-Controlled and Charge-Controlled Memristor
}

\author{
Qiang Guo, Weicheng Gu \& Zhikuo Tao \\ College of Electronic Science and Engineering, Nanjing University of Posts and \\ Telecommunications, Nanjing 210003, China.
}

Keywords: Memristor, hysteresis, quadratic.

\begin{abstract}
As the fourth basic passive component, memristor has attracted more attention in recent years. The memristor can be divided into charge-controlled and flux-controlled types. In this paper, properties of quadratic flux-controlled and charge-controlled memristor are investigated. The $v$ - $i$ characteristics of memristor with different parameters and different frequencies were studied under sinusoidal bias of voltage. The results show that, pinched hysteresis loops were observed for all memristors with different parameters, and different input frequencies.
\end{abstract}

\section{Introduction}

In 1971, Leon Chua predicted that there should be a fourth fundamental passive circuit element according to symmetry arguments, which he called a memristor (short for memory resistor) [1]. Later on, he did further research on memristive system [2]. We already know that, connecting pairs of the four fundamental circuit variables: electric current $i$, voltage $v$, charge $q$ and magnetic flux $\varphi$, there are five different mathematical relations. The relation of current $i$ and voltage $v(\mathrm{~d} v=R \mathrm{~d} i)$ is determined from the definition of resistance. The relation of charge $q$ and current $i(\mathrm{~d} q=i \mathrm{~d} t)$ is determined from the definitions of these two variables. The relation of current $i$ and magnetic flux $\varphi$ $(\mathrm{d} \varphi=L \mathrm{~d} i)$ is determined from the definition of inductance. The relation of voltage $v$ and charge $q$ $(\mathrm{d} q=C \mathrm{~d} v)$ is determined from the definition of capacitance and the relation of magnetic flux $\varphi$ and voltage $v(\mathrm{~d} \varphi=v \mathrm{~d} t)$ is determined from Faraday's law of induction. The memristor provides a missing link between charge $q$ and magnetic flux $\varphi$, and the introduced memristance is defined as $\mathrm{d} \varphi=M \mathrm{~d} q$.

As we can see from the definition of memristor, the memristance of different memristors is determined by charge and magnetic flux. The basic characteristic of memristor is related to the curve $f(\varphi, q)=0$ in the plane of $\varphi-q$ or $q-\varphi$, so memristors have two types: flux-controlled and charge-controlled. For the charge-controlled memristor, the relation of charge and flux can be expressed as $\varphi_{m}(t)=\varphi_{m}\left(q_{m}(t)\right)$. The memristance is

$$
M\left(q_{m}(t)\right)=\frac{d \varphi_{m}\left(q_{m}(t)\right)}{d q_{m}(t)}
$$

and the memductance is

$$
W\left(q_{m}(t)\right)=\frac{1}{M\left(q_{m}(t)\right)}
$$

For the flux-controlled memristor, the relation of charge and flux can be expressed as $q_{m}(t)=$ $q_{m}\left(\varphi_{m}(t)\right)$. The memductance is

$$
W\left(\varphi_{m}(t)\right)=\frac{d q_{m}\left(\varphi_{m}(t)\right)}{d \varphi_{m}(t)}
$$

and the memristance is

$$
M\left(\varphi_{m}(t)\right)=\frac{1}{W\left(\varphi_{m}(t)\right)}
$$




\section{The physical model and HP's charge-controlled memristor}

In 2008, R. S. Williams et al. reported the first device of charge-controlled memristor [3]. The device is based on $\mathrm{TiO}_{2}$ and has a sandwiched structure. The device between two electrodes consist two parts, $\mathrm{TiO}_{2}$ and $\mathrm{TiO}_{2-x}$. For $\mathrm{TiO}_{2}$ material, oxygen vacancy acts as a donor, and provides carriers of electron, so the side of $\mathrm{TiO}_{2-x}$ shows low resistance. The most important property is the boundary shifting between $\mathrm{TiO}_{2}$ and $\mathrm{TiO}_{2-x}$ under certain bias voltage.

For the model given by R. S. Williams et al., the relation of current $i$ and voltage $v$ is

$$
v(t)=\left(R_{O N} \frac{w(t)}{D}+R_{O F F}\left(1-\frac{w(t)}{D}\right)\right) i(t)
$$

where $R_{\mathrm{ON}}$ is the resistance of $\mathrm{TiO}_{2-\mathrm{x}}, R_{\mathrm{OFF}}$ is the resistance of $\mathrm{TiO}_{2}, w(t)$ is the length of $\mathrm{TiO}_{2-\mathrm{x}}$, and $D$ is the length of the device. So the memristance can be expressed as

$$
M=R_{O F F}\left(1-\frac{\mu_{V} R_{O N}}{D^{2}} q(t)\right)
$$

where $\mu_{\mathrm{V}}$ is the average ion mobility.

The current is not in phase with voltage when a sinusoidal bias is applied. Meantime, clear pinched hysteresis loops are observed in both analytical and experimental results. The work of Williams et al. inspired extensive research in this field. Pershin et al. studied the behavior of spin memristor in the semiconductor spintronics devices [4]. Wang et al. studied the spin torque-related memristor [5]. At the same time, the physical device is difficult to realize, while the relevant circuit simulations have been widely concerned. Biolek et al. presented the micro SPICE model [6] while Yu et al. [7] and Sánchez-López et al. [8] have demonstrated the floating emulators of memristor.

\section{Models of quadratic flux-controlled and charge-controlled memristor}

In this paper, we study the properties of quadratic flux-controlled and charge-controlled memristor, and the $v-i$ and $M-v$ characteristics with different parameters and frequencies. For the flux-controlled and charge-controlled memristor studied in this paper, the memristance can be expressed as:

$$
\begin{gathered}
M(\varphi)=\alpha \varphi^{2}+\beta \varphi+\gamma \\
M(q)=\alpha q^{2}+\beta q+\gamma
\end{gathered}
$$

We can see that, $M(\varphi)$ or $M(q)$ is linear flux-controlled or charge-controlled when $\alpha=0$. Memristor of this type has been researched widely, so we focus on the quadratic type here. For the model described by equation (7) and (8), we investigate the characteristics of $v$-i with different $\alpha$, $\beta$ and $\gamma$, as well as the characteristics with different frequencies of applied voltage. At the same time, we also notice that $M(\varphi)$ or $M(q)$ is positive or negative with different $\alpha, \beta$ and $\gamma$, so the instantaneous power $p(t)\left(p(t)=i(t)^{2} M(t)\right)$ and energy $w(t)\left(w(t)=\int p(\tau) \mathrm{d} \tau\right)$ are positive or negative. Then the memristor (with $M<0)$ is not a basic passive device. For the memristor investigated here, the $M(\varphi)$ or $M(q)$ is always positive with chosen value of $\alpha, \beta$ and $\gamma$.

\section{Results and discussions}

Characteristics of flux-controlled memristor.The $v$-i relations of analytical results with different $\alpha, \beta$ and $\gamma$ are shown in Fig. 1 (a), (b) and (c), respectively. The input sinusoidal voltage is $v(t)=A \sin (\omega t+\varphi)\left(\mathrm{A}=1 \mathrm{~V}, \omega=62.8(f=10 \mathrm{~Hz})\right.$ and $\left.\varphi=0^{\circ}\right)$.

Fig. 1 (a) shows the $v$-i relations with $\beta=5, \gamma=1$ and different $\alpha(10,100,1000,10000$ and 100000). We can see that obvious pinched hysteresis behavior is observed when $\alpha=10, \alpha=100$ and $\alpha=1000$, and there are no significant difference between $\alpha=10$ and $\alpha=100$. We also notice that, the hysteresis loops shrink with increasing the value of $\alpha$. As shown in the figure, hysteresis behavior is almost disappear when $\alpha=10000$ and $\alpha=100000$. The $v$-i relations are shown in Fig. 1 (b) 


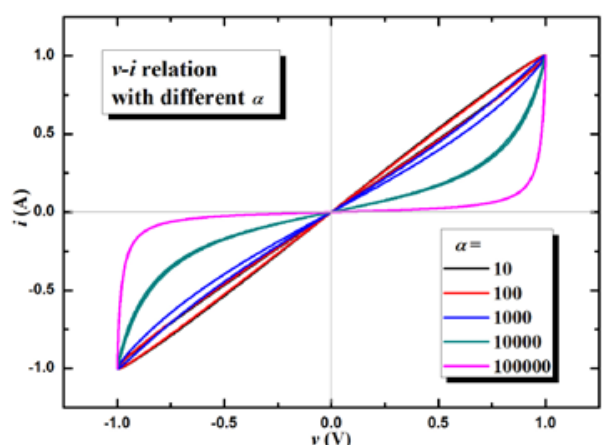

(a)

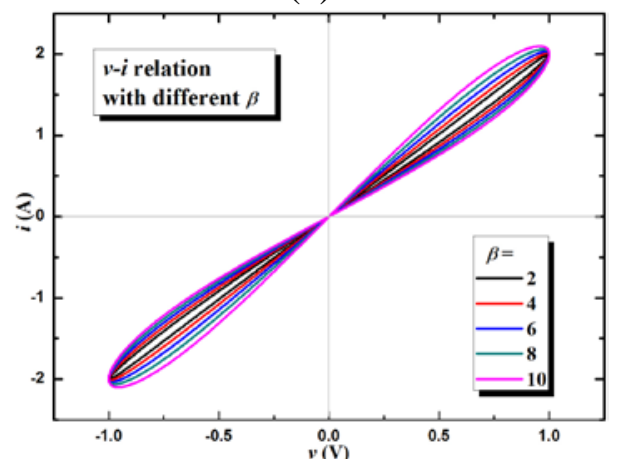

(b)

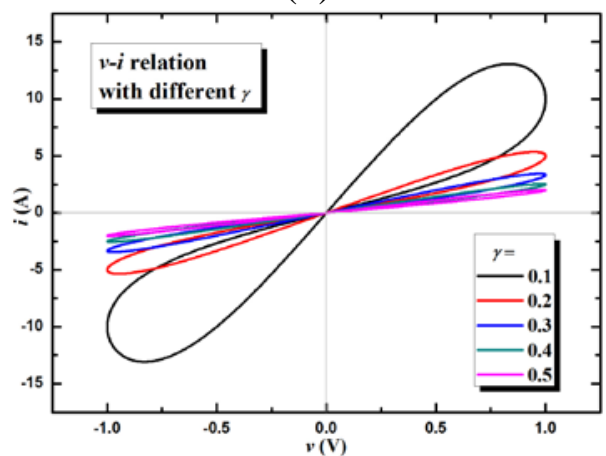

(c)

Fig. 1: The $v$-i relations with different $\alpha, \beta$ and $\gamma$.

(a) different $\alpha, \beta=5, \gamma=1$ (b) $\alpha=100$, different $\beta, \gamma=0.5$ (c) $\alpha=100, \beta=5$, different $\gamma$.

with $\alpha=100, \gamma=0.5$ and different value of $\beta$ (2, 4, 6, 8 and 10). It is clear that, the hysteresis behavior of $v$-i relations enhance significantly when $\beta$ increases from 2 to 10 . The $v$-i relations are shown in Fig. 1 (c) with $\alpha=100, \beta=5$ and different $\gamma(0.1,0.2,0.3,0.4$ and 0.5$)$. For all of these $v$-i relations, obvious hysteresis behaviors are observed, and the hysteresis behaviors decrease significantly with $\gamma$ increasing from 0.1 to 0.5 .

The $v-i$ and $M-v$ relations with different frequencies are shown in Fig. 2 (a) and (b). The parameters of this quadratic flux-controlled memristor are $\alpha=1, \beta=1$ and $\gamma=0.5$. Pinched hysteresis curves are obvious under different frequencies. More importantly, the pinched hysteresis loops shrink with increasing the frequency and tend to a straight line. This feature is same with the linear flux-controlled memristor. The memristances corresponding to input voltages under different frequencies are presented in Fig. 2 (b). The relation is a loop under certain frequency, and the range of memristance shrink gradually with the increase of frequency. With the frequency increasing toward infinity, the $M-v$ curve narrows into a line i.e. memristance is close to a constant. In this case, memristor is identical to a resistor with fixed value. 


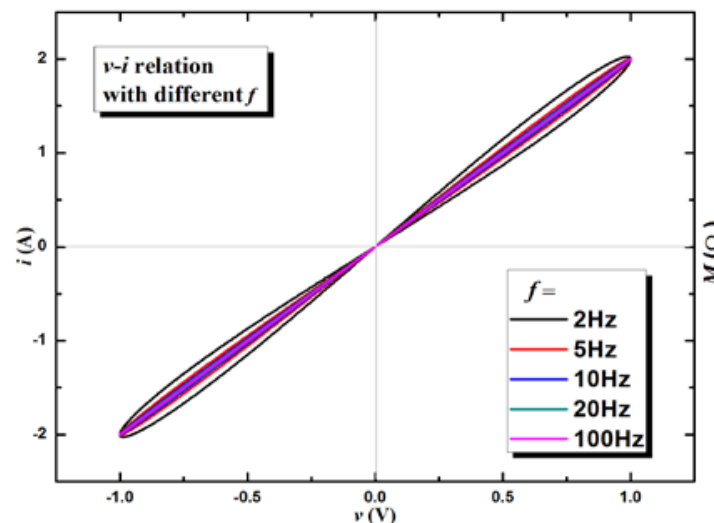

(a)

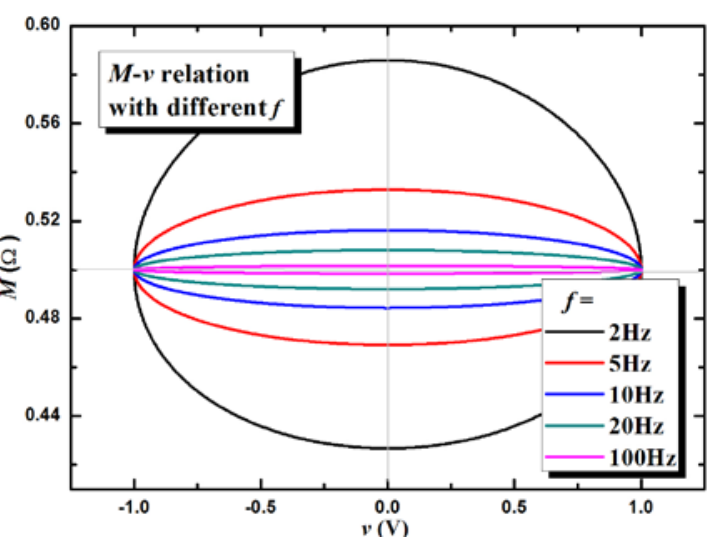

(b)

Fig. 2: (a) The $v$-i relations with different frequencies.

(b) The $M-v$ relations with different frequencies.

Characteristics of charge-controlled memristor.The analytical results of quadratic charge-controlled memristor obtained using same procedure are similar with the flux-controlled memristor discussed above.

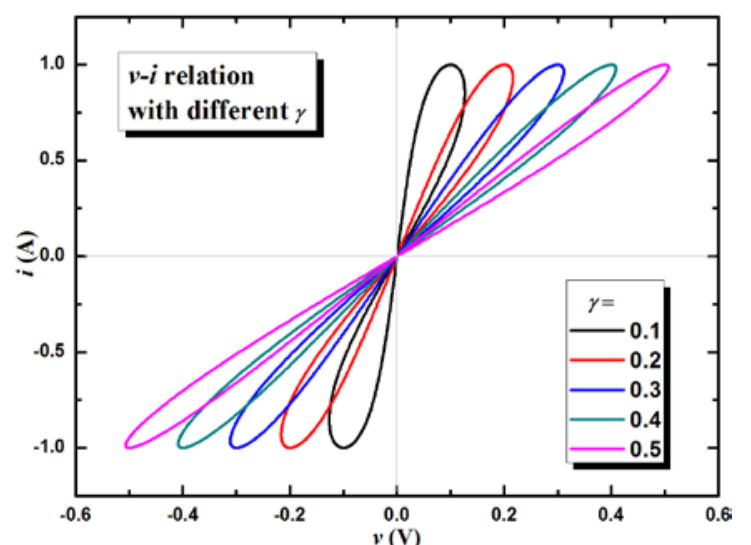

(a)

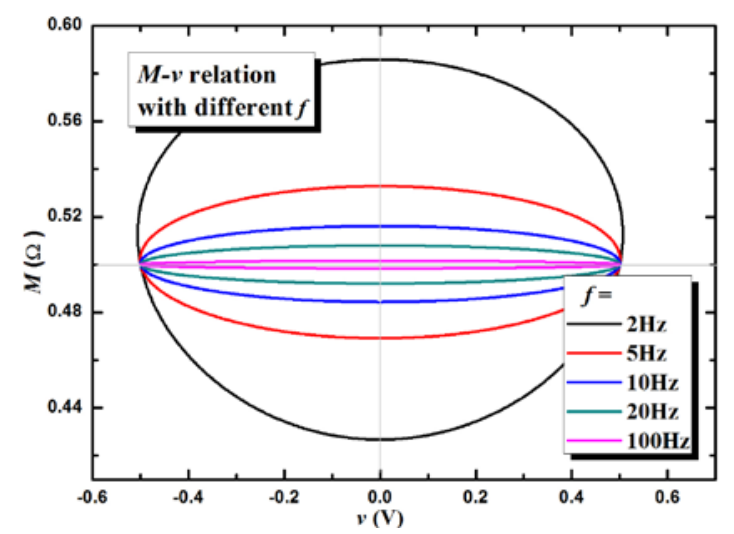

(b)

Fig. 3: (a) The $v-i$ relations with $\alpha=100, \beta=5$ and different $\gamma$.

(b) The $M-v$ relations with different frequencies.

Fig. 3 presents the typical $v$-i relations with different $\gamma$ (Fig. 3 (a)) and $M-v$ dependance with different input frequencies (Fig. 3 (b)).

\section{Conclusions}

In conclusion, properties of quadratic flux-controlled and charge-controlled memristor have been presented. Tipical $v$-i relations of analytical results with different parameter and with different input frequencies have been discussed. 


\section{Acknowledgements}

Email: guoqvip@163.com

\section{References}

[1] L. O. Chua. Memrister - the missing circuit element. IEEE Transactions on Circuit Theory, 1971, 18(5): 507-519.

[2] L. O. Chua and S. M. Kang. Memristive devices and systems. Proccedings of the IEEE, 1976, 64(2): 209-223.

[3] D. B. Strukov, G. S. Snider, D. R. Stewart and R. S. Williams. The missing memristor found. Nature, 2008, 453: 80-83.

[4] Yu. V. Pershin and M. Di Ventra. Spin memristive systems: Spin memory effects in semiconductor spintronics. Physical Review B, 2008, 78(11): 113309(1)-113309(4).

[5] X. Wang, Y. Chen, H. Xi, et al. Spintronic memristor through spin-torque-induced magnetization motion. IEEE Electron Device Letters, 2009, 30(3): 294-297.

[6] Z. Biolek, D. Biolek and V. Biolková. SPICE model of memristor with nonlinear dopant drift. Radio Engineering, 2009, 18(2): 210-214.

[7] D. S. Yu, H. H. Iu, A. L. Fitch, et al. A floating memristor emulator based relaxation oscillator. IEEE Transactions on Circuits and Systems - I: Regular Papers, 2014, 61(10): 2888-2896.

[8] C. Sánchez-López, J. Mendoza-López, M. A. Carrasco-Aguilar, et al. A floating analog memristor emulator circuit. IEEE Transactions on Circuits and Systems - II: Express Briefs, 2014, 61(5): 309-313. 